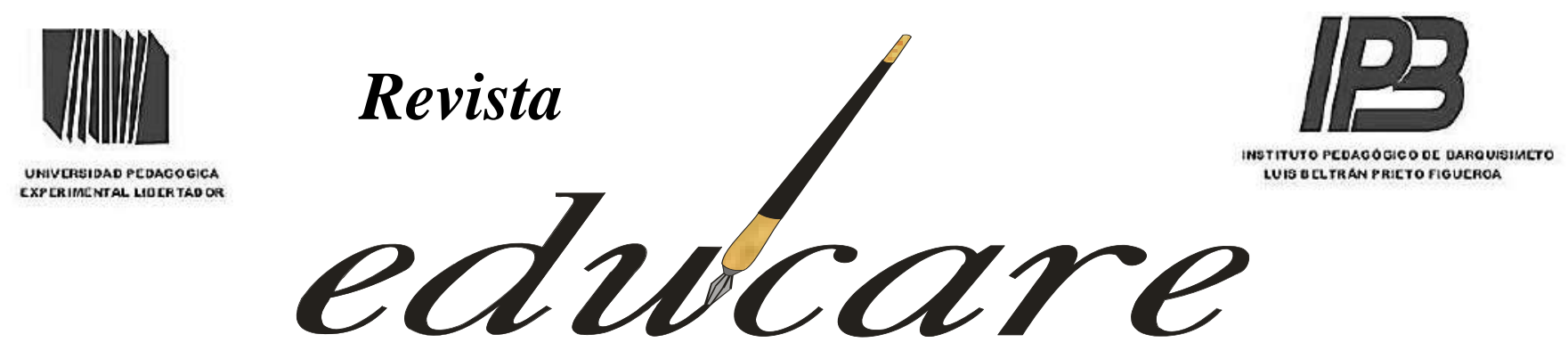

Órgano Divulgativo de la Subdirección de Investigación y Postgrado del Instituto Pedagógico de Barquisimeto "Luis Beltrán Prieto Figueroa"

Barquisimeto Estado Lara

\title{
ÁRBITROS COLABORADORES
}

NOMBRE DE ÁRBITROS

Dr. Alberto Rodríguez

Dr. Enrique Reyes

Dr. Henry Mujica

Dr. Luis Paradas

Dr. Manuel Ramírez

Dr. Marcos Flores

Dra. Adilia Flores

Dra. Ana Cecilia Reyes

Dra. Ana Hilda Castellón

Dra. Any Montero

Dra. Francia Becerra

Dra. Ismeray Páez

Dra. Lexy Mujica

Dra. María Lourdes Piñero

Dra. Mary Carmen Guedez

Dra. Milexa Sequera

Dra. Sandra Flores

\section{INSTITUCIÓN}

UPEL-IPB

UPEL-IPB

UPEL-IPB

UPEL-IPB

UPEL-IPB

UPEL-IPB

UPEL-IPB

UPEL-IPB

UPEL-IPB

UPEL-IPB

UPEL-IPB

UPEL-IPB

UPEL-IPB

UPEL-IPB

UPEL-IPB

UPEL-IPB

UPEL-IPB

UPEL-IPB 
Dra. Yarines Perdomo

Dra. Zaida Salazar

Msc. Patricia Quiroga

Prof. José Antonio Ladino

Prof ${ }^{a}$. Elvia Monsalve

Prof a . Francis González

Prof ${ }^{a}$. GiulianaFarci

Prof ${ }^{a}$. Liliam Alvarado

Prof ${ }^{a}$. Adlih Carolina González

Prof. Jesús Aranguren

Prof $^{\mathrm{a}}$. Catalina Betancourt

Dr. Amilcar Arenas

Prof ${ }^{\mathrm{a}}$. Ana Cristina Bolívar

Prof ${ }^{a}$. Zoraida Paredes

Msc. David González

Dr. Wilmer Chávez

Dra. Beatriz Veracoechea

Dr. José Rafael Prado

Dra. Dayli Quiva

Dr. José Ortiz

Dra. Omaira Oñate

Prof ${ }^{\mathrm{a}}$. Rhadis García de García

Prof ${ }^{a}$. Yvel Palacios

Dr. José Amador Sánchez Carreño

Lcdo. Samir El Hamra

Prof. Antonio Vera

Prof. José Gregorio Olivero León

Prof ${ }^{a}$. María Carrera

Prof ${ }^{\mathrm{a}}$. Marimily Segura

Dr. Kleeder Bracho

Dr. José de Carvalho

Dr. Alejandro Díaz M.
UPEL-IPB

UPEL-IPB

UPEL-IPB

UPEL-IPB

UPEL-IPB

UPEL-IPB

UPEL-IPB

UPEL-IPB

UPEL-IPC

UPEL-IPC

UPEL-IPC

UPEL-IPMACARO

UPEL-IPREM

UPEL-MARACAY

UCLA

UCLA

UCLA

ULA

UNERMB

Universidad de Carabobo

Universidad de Carabobo

Universidad de Carabobo

Universidad de Carabobo

UDO

UC

Luz-Punto Fijo

LUZ-Punto Fijo

LUZ-Punto Fijo

Universidad José Antonio Páez

Ministerio del Poder Popular para la Educación

Universidad de San Paulo-Brasil

Universidad de Concepción -Chile 
Dr. Napoleón Murcia

Dr. Oscar Ranulfo Ayala Aragón

Dr. René Pedroza Flores

Dra. Cristina López

Dra. Lorena Cruz

Dra. María Soledad Loutayf

Dra. Norma Ivonne González Arratia

Ing. Edgar Serna

Lcda. Marjorie Rodríguez

Lcdo. Luis Humacata

Prof. Carlos Meléndez Tamayo

Prof. Jorge H. Aristizábal Zapata

Prof. Juan Jesús Velasco

Prof. Luis Puga
Universidad de Caldas. Manizales

Colombia

Universidad Autónoma Tomás Frías

(Potosí, Bolivia)

Universidad Autónoma del Estado de

México (UAEM)

Centro Universitario del Sur Universidad

de Guadalajara

Universidad Nacional de Cuyo -

Argentina

Facultad de Ciencias Exactas de la

UNAS- Argentina

Universidad Autónoma del Estado de

México (UAEM)

Instituto Tecnológico Metropolitano-

Medellín Colombia

Universidad del Norte. Barranquilla

Colombia

Universidad Nacional de Luján -

Argentina

Universidad Técnica de Ambato. Ecuador

Universidad del Quindío. Colombia

Universidad Autónoma del Estado de

México (UAEM)

Universidad Tecnológica Equinoccial-

Ecuador 